\title{
كلمات فرنسية من أصل عربي
}

\section{French words from Arabic}

\section{Khaled Moustafa ${ }^{1}$ خالد مصطفى}

عند الإفطار، إذا طلبت باللغة الفرنسية طاساً من القهوة، مع أو بدون سكر، أو عصير برتقال أو شراب مشمش، وأنت جالس على أريكة أو سرير، مرتدياً قميصاً أبيضَ وسروالاً أسودَ، ومتصفحاً مجلتك المفضلة عن الخوارزميات أو الكيمياء أو أضرار الكحول كما يؤكدها طبيب متخصص، فستكون قد استخدمت خمس عشرة كلمة فرنسية من أصل عربي (أو معرّة). ثم، إذا ذهبت إلى السوق لشراء بعض البطيخ أو السبانخ، مع بعض الكمون، والسماق، والكركم، أو الزعفران، وعدت إلى البيت بالحافلة بتعرفة مخفضّة، أو مشياً على الرصيف أو القطران، فستكون قد استخدمت عشر كلمات إضافية من أصل عربي". فيكون المجموع خمساً وعشرين كلمة من أصل عربي في نص فرنسي من حوالي مئة كلمة فرنسية. كلمات مفتاحية: اللغة العربية وتأثثر ها، مفردات عربية في لغات أجنبية، اللغة الفرنسية، كلمات فرنسية من أصل عربي.

هنالك حوالي سبعة آلاف لغة محكية في العالم اليوم (1)، تتأثز كل منها وتؤثز في الأخرى، وينتج عن ذلك كلمات هجينة وأخرى جديدة. ومن بين اللغات التي نأثرت باللغة العربية بشكل كبير، هي اللغة الفرنسية (2-4) وذلك نتيجة التواصل المباشر بين العرب والفرنسيين، أو الاحتكاك غير المباشر من خلال لغات أخرى وسيطة كالإسبانية والإيطالية والبرتغالية. ويوضح المثال التالي مدى تأثير اللغة العربية على اللغة الفرنسية من خلا النسبة الكبيرة لعدد الكلمات الفرنسية من أصل عربي (أو معربة، من أصل فارسي أو تركي...)، مُشار إليها بالغامق، وكثير منها موجود أيضاً في لغات أخرى، غير الفرنسية، بألفاظ مشابهة تقربياً:

Au petit déjeuner, si vous commandez une tasse de café, avec ou sans sucre, et un jus d'orange ou du sirop d'abricot, en vous asseyant sur un sofa ou sur un matelas, en portant votre chemise blanche et votre saroual noir, et en lisant votre magazine préféré sur les algorithmes, la chimie ou les effets de l'alcool sur la santé rapportés par un toubib spécialiste, vous utiliserez une quinzaine de mots français d'origine arabe. Si vous passez ensuite au souk ou au magasin pour acheter de la pastèque ou de l'épinard, avec du cumin, du sumac, du curcuma, ou du safran, et vous rentrez chez vous en bus à un tarif réduit ou en marchant sur le récif ou le goudron, vous en utiliserez encore davantage.

\footnotetext{
${ }^{1}$ Editor of the Arabic Science Archive (arabixiv.org). Correspondence: Khaled.moustafa@arabixiv.org
} 


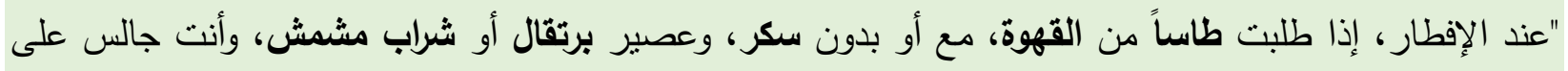

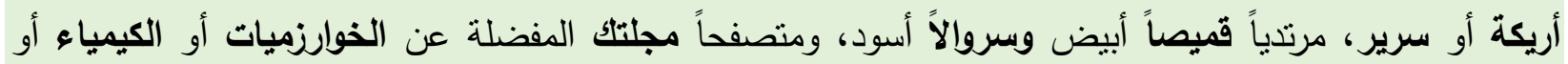

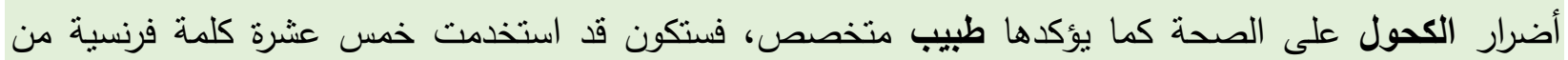

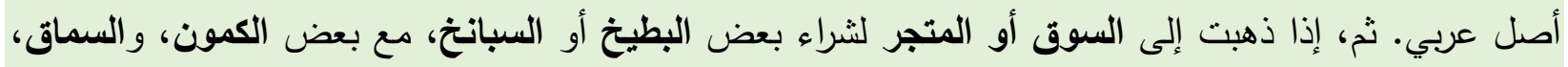

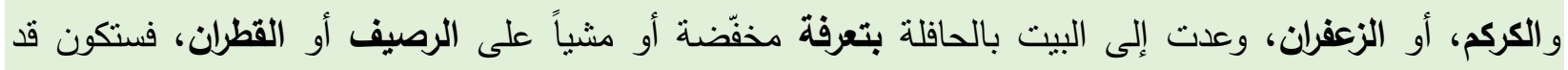
استخدمت عشر كلمات إضافية في اللغة الفرنسية من أصل عربي".

وهكذا، يكون المجموع 25 كلمة تقريباً من أصل عربي في نص من حوالي 100 كلمة فرنسية.

وفي هذا الصدد، يقول أحد اللغويين الفرنسين(3) "إن اللغة العربية هي من اللغات الرئيسية التي ثُدين لها اللغة الفرنسية بكثير من المفردات المُستعارة، وتحتوي منها على ما يقارب ضعفي ما تحتويه من

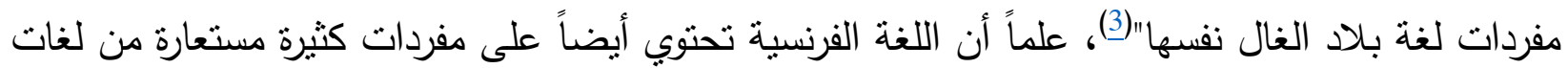
أخرى كاللاتينية والإيطالية والإسبانية والإنكليزية، إلخ.

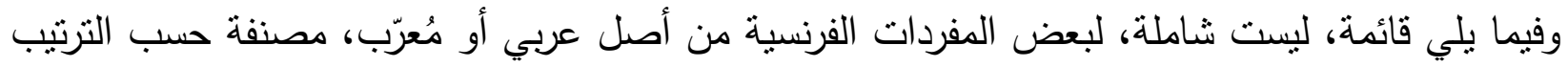

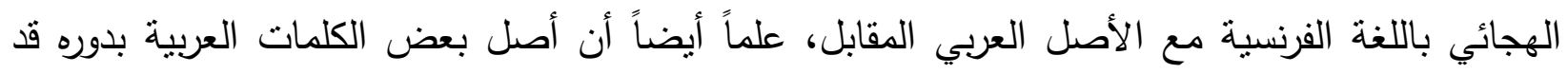
يكون فارسياً أو تركياً معرباً أو عربياً بلهجة أهل الهن المغرب العربي، ولكنه أصبح من مفردات اللغة الفرنسية

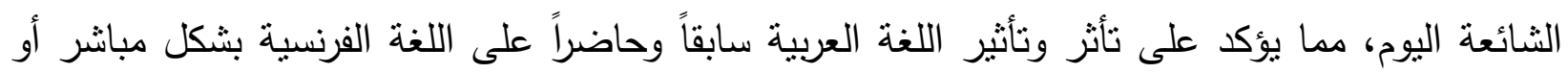
غير مباشر · 
جدول (1). كلمات فرنسية مع لفظها بالفرنسي بين قوسين (العمود الأيسر) و أصلها العربي أو المعرّب (العمود الأيمن).

\begin{tabular}{|c|c|}
\hline الكلمة الفرنسية ولفظها & الأصل العببي أو المعرب \\
\hline بالعبي بين قوسين & ل للكلمة الفرنسية \\
\hline abricot (أبريكو) & البرقوق، مشمش \\
\hline adobe (أدوب) & الطوب (لبنة، آجرة) \\
\hline alcad (الكاد) & قاضي \\
\hline alcali (الكالي) & القلي (مقلي نبات لانتاج الصودا) \\
\hline alcazar (الكازار) & القصر \\
\hline alchimie (أشيمي) & الكيمياء، الخيمياء \\
\hline alcool (ألكول) & 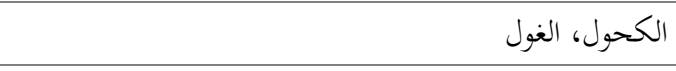 \\
\hline alcôve (ألكوف) & القبة \\
\hline alezan (أليزان) & الحصان \\
\hline 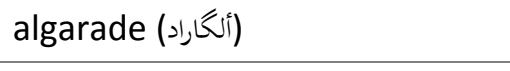 & الغارة \\
\hline algèbre (ألجيبر) & الجبر \\
\hline algorithme (ألكوريتم) & المستعرب) \\
\hline alidade (أليداد) & 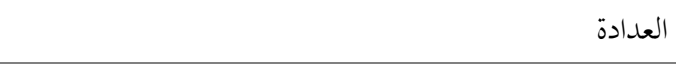 \\
\hline alkaline (ألكالين) & قلوي \\
\hline almanach (المناك) & المناخ (تقويم، روزنامة) \\
\hline amalgame (أمالخام) & الملْغنم \\
\hline ambre (آمبر) & عنبر \\
\hline amiral (أميرال) & أمير البحر \\
\hline arabette "أرابيت" & أرابيت: نبات صغير شاهده الرومان نامياً بكثرة في بلاد العرب \\
\hline (الاسم لعلمي (Arabidopsis thaliana) & عند تسميتهم له بهذا الاسم ع \\
\hline arrobe (أرّب) & الربع (وحدة قياس قديمة تعادل ما بين 11 إلى 15 كغ، ورمزها \\
\hline 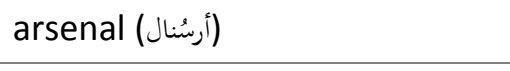 & دار الصناعة \\
\hline artichaut (آرتيشو) & الخرشوف \\
\hline assassin (أسّاسَان) & حشاش (ثم أخذت معنى قاتل أو بحرم باللغة الفرنسية) \\
\hline athanor (أتّانور) & التنّور \\
\hline aubergine (أوبرجين) & باذبخان (فارسية) \\
\hline azerole (آزيرول) & الزعرور \\
\hline azimuth, azimuth, zénith (آنيموت) & السمت (ابتاه رأسي أو عمودي أو علوي، ويقابله النظير) \\
\hline azur (آزور) & 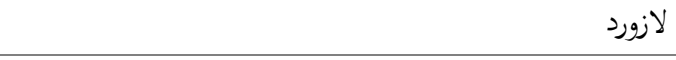 \\
\hline
\end{tabular}


Arabic Science Archive (arabixiv.org)|2018

\begin{tabular}{|c|c|}
\hline الكلمة الفرنسية ولفظها & الأصل العببي أو المعرب \\
\hline ب بالعربي بين قوسين & للكلمة الفرنسية \\
\hline babouche (بابوش) & بابوج (حذاء) \\
\hline banane (بانان) & بنان (تشبيه ثمار الموز بأصابع اليد) \\
\hline baraka (باراكا) & بركة \\
\hline barda (باردا) & بردعة (جهاز الحصان أو مؤنة الجندا) \\
\hline barkhane (برخان) & برخان (كثيب رملي مرتفع) \\
\hline basane (بازان) & بطانة (بلاس) \\
\hline bésef, béseff, bézef (ييزاف) & "بالزاف" (كثير) \\
\hline 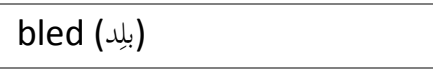 & بلد، بلاد \\
\hline bordj (برج) & برج \\
\hline bougie (بوجي) & شععة، نسبة إلى مدينة بحاية في الجزائر \\
\hline cabas (كابا) & قُقَة (سلة، حقيبة) \\
\hline cadi ou kadi (كادي) & قاضي \\
\hline cador (كادور) & قادر \\
\hline café (cahoua, cahva) (كافه) & 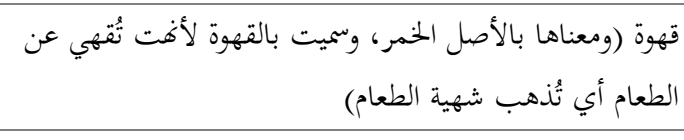 \\
\hline caïd (كايد) & قائد \\
\hline calibre (كاليب) & قالب \\
\hline camelot (كاملو) & خمل (خمل الصوف أو السجاد) \\
\hline camphre (كامفر) & كافور \\
\hline candi (كاندي) & قند (عصارة قصب السكر إذا جمد) \\
\hline carafe (كاراف) & غرف (الماء) \\
\hline carat (كاراه) & قيراط (مقياس لدرجة نقاوة الذهب) \\
\hline carmin (كارمان) & قرمز \\
\hline caroube (كاروب) & 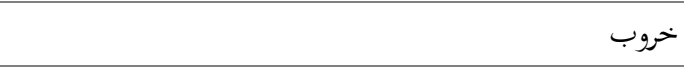 \\
\hline carthame (كارتام) & قرطم \\
\hline chadouf (شادوف) & شادوف (جهاز يستخدم في الري) \\
\hline chemise (انُميز) & قميص \\
\hline chergui (شيركي) & شرقي (رياح شرقية، هواء شرقي، نسيم شرقي) \\
\hline chérif (شيريف) & شريف ر \\
\hline chiffre (شيفر) & صفر \\
\hline chimie (شيمي) & كيمياء \\
\hline chott (شوط) & شط \\
\hline chouia (شُويًا) & شوية (تصغير "شيء": قليل) \\
\hline
\end{tabular}


Arabic Science Archive (arabixiv.org)|2018

\begin{tabular}{|c|c|}
\hline الكلمة الفرنسية ولفظها & الأصل العربي أو المعرب \\
\hline 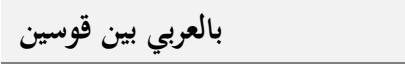 & للكلمة الفرنسية \\
\hline coton (كوتون) & قطن \\
\hline couffin (كوفان) & قفة \\
\hline cramoisi (كراموزي) & قرمزي \\
\hline cumin (كُمَان) & كمون \\
\hline curcuma (كُرُْمَا) & كُركم \\
\hline dahir (داهير) & ظهير (مرسوم ملكي) \\
\hline dinar (دينار) & دينار (عملة ذهبية) \\
\hline درهم) Dirham/dirhem & درهم (عملة فضية) \\
\hline divan (ديفان) & ديوان \\
\hline djébel (جييل) & جبل \\
\hline djellaba/djellabah (جيّابا) & قلابية أو جلابية (ثوب عربي) \\
\hline 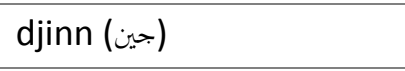 & جن \\
\hline douane (دُوان) & ديوان \\
\hline drogman (دروخمان) & ترجمان (مترجم) ت \\
\hline échec (إيشيك) & من الفارسية شاه (شيخ، ملك) ومنه لعبة الشطرنج \\
\hline éfrit (إيفريت) & عفريت ع \\
\hline élixir (إليكسير) & الكسير (من كَسَرَ حجر الفلاسفة) \\
\hline émir (إيمير) & أمير (آمر) \\
\hline épinard (إيبينار) & سبانخ (من الفارسية، اسبناج) \\
\hline estragon (إيستراجون) & طرخون \\
\hline fakir ou faquir (فاكير) & فقير \\
\hline farde, fardeau (فارد، فاردو) & فرد (صندوق، ثقيل) \\
\hline $\begin{array}{l}\text { flouze, flouse, flous, fric } \\
\text { (فلوز، فلوز، فلوس،فريك) }\end{array}$ & 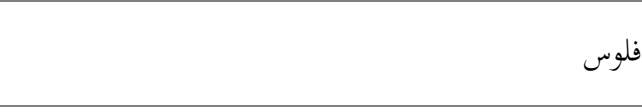 \\
\hline fondouk (فوندوك) & فندق \\
\hline gabelle (كابيل) & قبالة (ضريبة، أو أداء شيء مقابل مبلغ معين) \\
\hline gazelle (عازيل) & غزال، غزالة غ غ \\
\hline gerboise (جيربواز) & جربوع أو يربوع (حسب اللهجة العربية) (حيوان قارض فوق \\
\hline ghazel ou ghasel (كَازل) & غزل (مدح، عند الجديث عن الشعر "شعر غزل) \\
\hline gingembre (جانْامبر) & 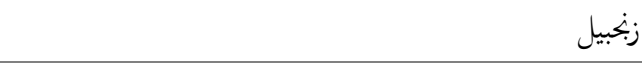 \\
\hline girafe (جيراف) & زرافة \\
\hline goudron (كُودرون) & 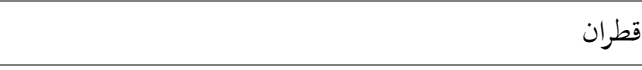 \\
\hline
\end{tabular}


Arabic Science Archive (arabixiv.org)|2018

\begin{tabular}{|c|c|}
\hline الكلمة الفرنسية ولفظها & الأصل العببي أو الدعرب \\
\hline ب بالعربي بين قوسين & للكلمة الفرنسية \\
\hline \multirow{2}{*}{ goule (كؤل) } & غول (الحية) أو "الشيطان الذي يأكل الناس"، ومنه قول العرب \\
\hline & "الغول والعنقاء والخل الوفي من المستحيلات" \\
\hline goum (كؤم) & قوم \\
\hline 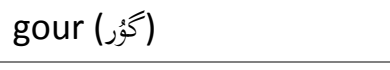 & غور \\
\hline guitar (كِيتار) & قيثار أو قيثارة \\
\hline hadith (هاديت) & حديث \\
\hline haïk (هايك) & حايك، خياط \\
\hline halva (هالفا) & حلوى، حلاوة \\
\hline hamada (هاماد) & ممادى (برية أو سهل صحراوي) \\
\hline hammam (هامام) & متمّام \\
\hline haram (هارم) & حرام (خطية) \\
\hline harira (هاريرا) & حريرة (شوربة، حساء) \\
\hline harissa (هاريسا) & هريسة (عجينة مهروسة) \\
\hline hasard (آزار) & زهر (تطورت إلى حظ أو صدفة) \\
\hline hedjaz ou hejaz (هيجاز) & الحجاز (مكة والمدينة وما حولمما) \\
\hline hégire (هيجير) & هجرة \\
\hline henné (إِيّْ) & حنة (نسبة إلى نبات الحناء) \\
\hline 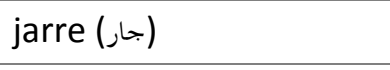 & جرة \\
\hline jellaba, djellaba (جيلاب) & جلابية أو قلابية (ثوب عربي) \\
\hline 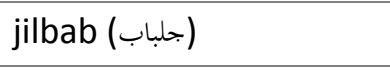 & جلباب \\
\hline 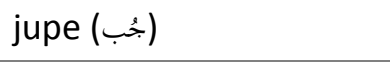 & جبة (تنورة) \\
\hline kadi (cadi) (كادي) & قاضي \\
\hline kali (كالي) & قلي (نبات يستخلص منه الصود) \\
\hline kan ou khan (كان) & خان \\
\hline keffieh (كيفيه) & كوفية (غطاء الرأس) \\
\hline kermès (كيميز) & قرمز \\
\hline ketmie (كيتمي) & ختمية أو خطمية (نبات زينة ذو أزهار ملونة كبيرة) \\
\hline khalife (calife) (كاليف) & خليفة (حاكم) \\
\hline khamsin (كامسان) & خماسين (رياح صحراوية) \\
\hline khôl (kuhl, kohol) (كوهل) & كحل (صبغة العين) \\
\hline kibla (qibla) (كيب) & قبلة (اتحاه) \\
\hline kif-kif (kifkif) (كيف-كيف) & نفس الشيء \\
\hline koubba (qubba) (كوبّ) & قُبَة \\
\hline
\end{tabular}


Arabic Science Archive (arabixiv.org)|2018

\begin{tabular}{|c|c|}
\hline الكلمة الفرنسية ولفظها & الأصل العبي أو المعرب \\
\hline بالعربي بين قوسين & للكلمة الفرنسية \\
\hline ksar (كسار) & قصر \\
\hline lemon (ليمون) & ليمون \\
\hline lilas (لِيلا) & نبات الليلك \\
\hline litham (ليتام) & ل ل لثام \\
\hline loukoum (لوكوم) & لقم (راحة الحلقوم) \\
\hline maboul (mahbul) (مابول) & مهبول \\
\hline machrek ou machreq (ماشريك) & مشرق (الشرق) \\
\hline $\begin{array}{l}\text { magasin (et magazine) } \\
\text { ماكازان (و ماكازين) }\end{array}$ & مخزن (و بحلة) \\
\hline maghreb (ماكربب) & 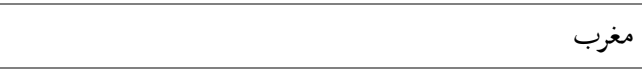 \\
\hline mahonne (ماهون) & 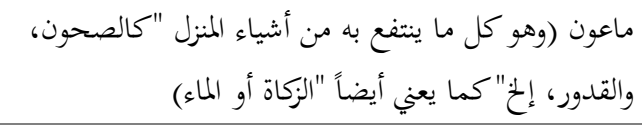 \\
\hline $\begin{array}{l}\text { maroquin, maroquinerie } \\
\text { (ماروكان، ماروكينري) }\end{array}$ & مراكشي (وصف لصناعة الجلود، نسبة إلى مدينة مراكش في \\
\hline masser (ماسّيه) & 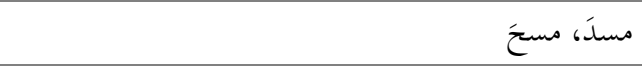 \\
\hline matelas (ماتلا) & مَطْرَحة (مكان الطرح أو الاستلقاء أو الجلوس) \\
\hline matraque (ماتراكs) & مطرقة \\
\hline mazout (مازوت) & معزول (نفاية، بقايا...) (من الروسية) \\
\hline méchoui (ميشوي) & 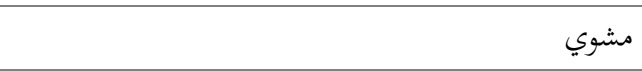 \\
\hline médersa, medersa (ميديرسا) & 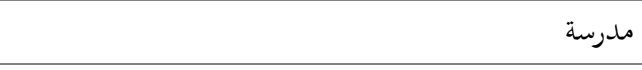 \\
\hline médina (ميدينا) & 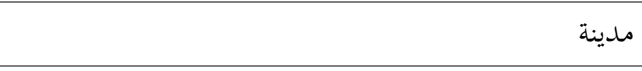 \\
\hline mihrab (ميهراب) & 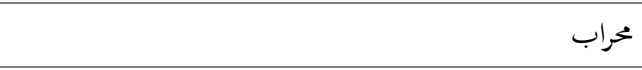 \\
\hline minaret (ميناريه) & 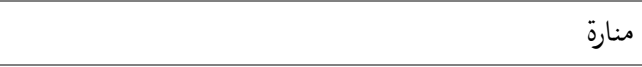 \\
\hline minbar (مانبار) & منبر \\
\hline mohair (موهير) & مُخيّر (مُلتقط، يُختار، يُخَيّر) \\
\hline muezzin (مُويزان) & مؤذن \\
\hline nabi (نابي) & نبي \\
\hline nadir (نادير) & نظير \\
\hline noria (نوريا) & ناعورة (يُستقى به الماء) \\
\hline orange (أورانج) & نارنج \\
\hline 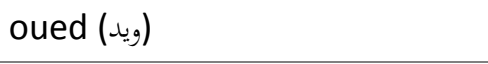 & واد \\
\hline $\begin{array}{l}\text { papegai, papegaut, perroquet } \\
\text { (بابيكي، بابيكو، بيروكيه) }\end{array}$ & ببغاء \\
\hline
\end{tabular}


Arabic Science Archive (arabixiv.org)|2018

\begin{tabular}{|c|c|}
\hline 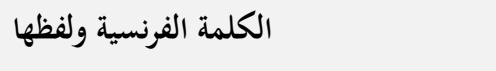 & الأصل العببي أو المعرب \\
\hline بالعربي بين قوسين & ل للكلمة الفرنسية \\
\hline pastèque (باستيك) & بطيخ \\
\hline qibla, kibla, kiblat (كيبل) & قِبلة \\
\hline quintal (كانتال) & قنطار (وحدة وزن تعادل ما يقارب 100 كغ) \\
\hline rabab, rebab (راباب، ريياب) & ربّابة \\
\hline ramdam, ramadan (رامدام، رامادان) & رمضان \\
\hline 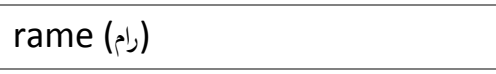 & رزمة \\
\hline raquette (راكيت) & راحة (اليد) \\
\hline razzia (غازيا، رازيا) & 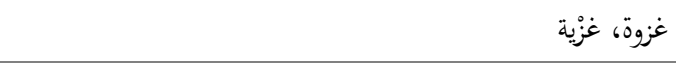 \\
\hline récif, rescif, ressif (ريسيف) & رصيف \\
\hline riad, riyad (رياد) & رياض (موضع يجتمع فيه الماء والخضرة أو العشب) \\
\hline risque (ريسك) & 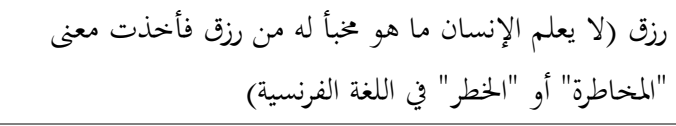 \\
\hline sacre, sacret (ساكر، ساكريه) & صقر \\
\hline safari (سافاري) & سفري (نسبة إلى السفر) \\
\hline safran (سافران) & زعفران \\
\hline sahara (ساهارا) & صحارى (جمع صحراء) \\
\hline 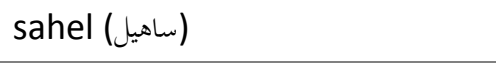 & ساحل \\
\hline $\begin{array}{l}\text { sakieh, sakyeh, saki } \\
\text { (ساكييه، ساكيه، ساكي) }\end{array}$ & ساقية \\
\hline salamalec (سالاماليك) & سلام عليك! (إدغام من السلام عليكم) \\
\hline $\begin{array}{l}\text { saroual, sarouel ou seroual } \\
\text { (ساروال، سارويل، سيروال) }\end{array}$ & 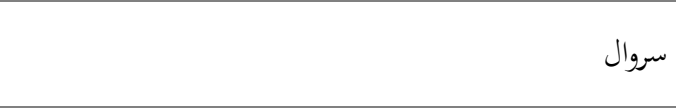 \\
\hline 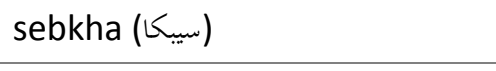 & سبخة س س س س س \\
\hline simoun (سيمون) & السموم (رياح السموم، صحراوية جافة) \\
\hline sirop (سيرو) & شراب \\
\hline sofa (سوفا) & صوفا (من الصوف) \\
\hline soude (سؤد) & من سواد رماد الروثا (نبات) عند حرقه للحصول على "الصود" \\
\hline 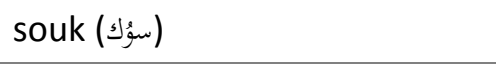 & 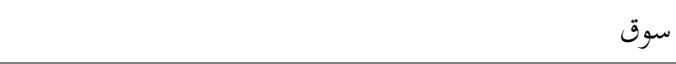 \\
\hline 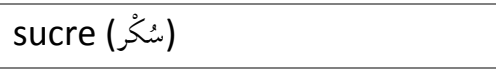 & سكر \\
\hline sultan (سُشْتان) & سلطان \\
\hline sumac (سمُكاك) & سماق \\
\hline taboulé (تابوليه) & 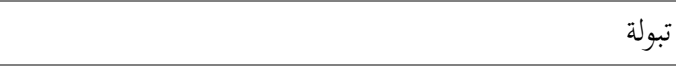 \\
\hline tabouret, tabour (تابوريه، تابور) & طمبور \\
\hline
\end{tabular}


Arabic Science Archive (arabixiv.org) $\mid 2018$

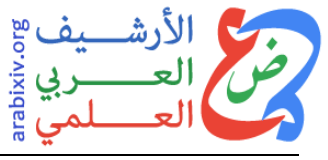

\begin{tabular}{|c|c|}
\hline الكلمة الفرنسية ولفظها & الأصل العربي أو المعرب \\
\hline بالعربي بين قوسين & 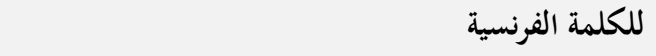 \\
\hline tajine (تاجين) & طاجين (أكلة مغاربية) \\
\hline talc (تالك) & طلق (مسحوق) \\
\hline tamarin (تاماران) & تمر هندي \\
\hline tambour ou tambourin & \\
\hline 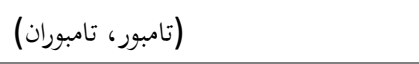 & طبل \\
\hline tare, tarer (تارر، تاريه) & طرّح (إنقاص وزن أو قيمة معينة من قيمة أخرى) \\
\hline tarif, tariff (تاريف) & تعرفة \\
\hline tasse (تاس) & طاس (وعاء يُشرب به) \\
\hline tcharchaf (شارشاف) & شرشرف (تركية) \\
\hline toubib (توبيب) & طبيب (حكيم) \\
\hline vizir (wazir) (وزير) (فيزير) & 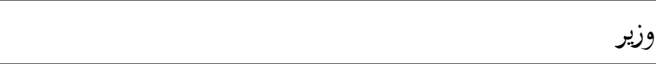 \\
\hline wilaya (vilayet) (ولايا) (فيلاييه) & ولاية (منطقة، مصر: مفرد أمصار) \\
\hline zain (زين) & زين (جميل، حسن) \\
\hline zénith (زينيت) & مشتقة من سمت (ابتحاه رأسي، عمودي، علوي، يقابله النظير) \\
\hline zéro (زيرو) & صفر $\quad$ ص \\
\hline
\end{tabular}

بالمقابل، تحتوي اللغة العربية بدورها على مفردات كثثرة أيضاً من لغات أخرى، كالتركية والفارسية والإنكليزبية والفرنسية واليونانية القديمة (الإغريقية) 5.

\section{References}

1. Lewis, M.P., G.F. Simons, and C.D. Fennig, Ethnologue: Languages of the world. Vol. 9. 2009: SIL international Dallas, TX. https://www.ethnologue.com

2. Guemriche, S., Dictionnaire des mots francais d'origine arabe. 2007: Éd. du Seuil. France.

3. Pruvost, J., Nos ancêtres les Arabes, ce que notre langue leur doit. 2017: JC Lattès. France.

4. Řehořová, J., Emprunts arabes en français. 2007, Masarykova univerzita, Pedagogická fakulta.

5. Moustafa, K. "غاية الأرب في معرفة أصل كلام العرب", Arabic Science Archive (arabixiv), 2018, doi: https://dx.doi.org/10.17605/osf.io/bemg8 